\title{
Studies on the Effects of Test Gas on the Flow Field around Large Angle Blunt Cone Flying at Hypersonic Mach Number
}

\author{
By Gopalan JaGadeesh,${ }^{1)}$ Kanderpallt Nagashetty, ${ }^{1)}$ K. P. Jaganntha Reddy, ${ }^{1)}$ \\ Mingyu SuN ${ }^{2)}$ and Kazuyoshi TAKAYAMA ${ }^{2)}$ \\ ${ }^{1)}$ Department of Aerospace Engineering, Indian Institute of Science, Bangalore, India \\ ${ }^{2)}$ Shock Wave Research Center, Institute of Fluid Science, Tohoku University, Sendai, Japan
}

(Received November 26th, 2001)

\begin{abstract}
The effect of the test gas on the flow field around a $120^{\circ}$ apex angle blunt cone has been investigated in a shock tunnel at a nominal Mach number of 5.75. The shock standoff distance around the blunt cone was measured by an electrical discharge technique using both carbon dioxide and air as test gases. The forebody laminar convective heat transfer to the blunt cone was measured with platinum thin-film sensors in both air and carbon dioxide environments. An increase of 10 to $15 \%$ in the measured heat transfer values was observed with carbon dioxide as the test gas in comparison to air. The measured thickness of the shock layer along the stagnation streamline was $3.57 \pm 0.17 \mathrm{~mm}$ in air and $3.29 \pm 0.26 \mathrm{~mm}$ in carbon dioxide. The computed thickness of the shock layer for air and carbon dioxide were $3.98 \mathrm{~mm}$ and $3.02 \mathrm{~mm}$, respectively. The observed increase in the measured heat transfer rates in carbon dioxide compared to air was due to the higher density ratio across the bow shock wave and the reduced shock layer thickness.
\end{abstract}

Key Words: Blunt Cones, Hypersonic Shock Wave

\section{Introduction}

Viscous- and entropy-swallowing effects are some of the dominant features of the hypersonic flow regime. Recent$1 y,{ }^{1,2)}$ experimental heat transfer measurements on the fore body of $120^{\circ}$ blunt cones have been correlated in terms of the viscous interaction parameter and shock density ratio. The heat transfer correlation function $\chi$ is given by

$$
\chi=\operatorname{St}\left(\rho_{2} / \rho_{\infty}\right)^{2 / 3} / M_{\infty}\left(C^{*} / R e_{\infty n}\right)^{0.5}
$$

where $R e_{\infty}$ is the Reynolds number based on the nose radius of the blunt cone model, $C^{*}$ is the Chapman-Rusbin factor given by $\left(\mu^{*} T_{\infty} / \mu_{\infty} T^{*}\right)$, and $\mu^{*}$ is the reference viscosity calculated at the reference temperature $T^{*}(K)=$ $\left(T_{02} / 6\right)\left(1+3 T_{\mathrm{w}} / T_{02}\right)$. The starred quantities are the reference values, and the subscripts ' $w$ ' and ' 02 ' indicate the wall and the stagnation conditions, respectively. The exponent of the density ratio is attributed to the bluntness effect, body geometry, and flow conditions. It was observed that the heat transfer measurements correlated well irrespective of the angle of attack. A typical variation of $\chi$ expressed in terms of wetted surface length $s / R_{\mathrm{n}}$ for the blunt cone model with a slightly blunted corner is shown in Fig. 1. It appears that $\chi$ is very sensitive to the variation of $\gamma$, and slight differences in the correlated heat transfer distributions were observed for carbon dioxide compared to air as the test gas. However, it is important to remember that this correlation does not encompass all physics and chemistry of the flow conditions, and more experiments are necessary for including the effect of variation of $\gamma$ on the heat transfer cor-

(c) 2002 The Japan Society for Aeronautical and Space Sciences relations. The present experimental study, which uses the Indian Institute of Science (IISc) shock tunnel HST2, aims at further understanding the effect of test gas other than air, such as carbon dioxide, on the flow field around a $120^{\circ}$ apex angle blunt cone flow field at Mach 5.75.

In recent times spurred on by the success of a Mars pathfinder mission, interest has been renewed in blunt-body entry vehicles and aerobrake configurations such as $70^{\circ}$ and $60^{\circ}$ semi-apex angle blunt cones. This interest has led to several significant experimental studies of large angle blunt body flows ${ }^{3,4)}$ at hypersonic Mach numbers. The visualization of a detached shock wave that forms ahead of the blunt body in hypersonic flight is an important aspect of hypersonic research because the shock standoff distance is a convenient parameter for validating any analytical or numerical models before using them for predicting the other flowfield

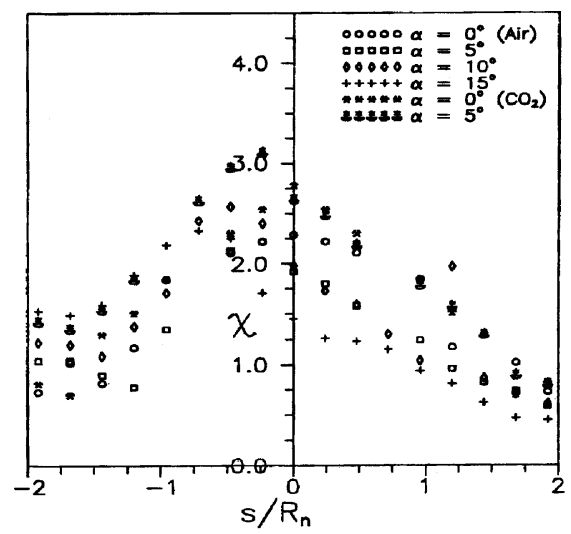

Fig. 1. Convective heat transfer correlation for the $120 \mathrm{deg}$. blunt cone. 


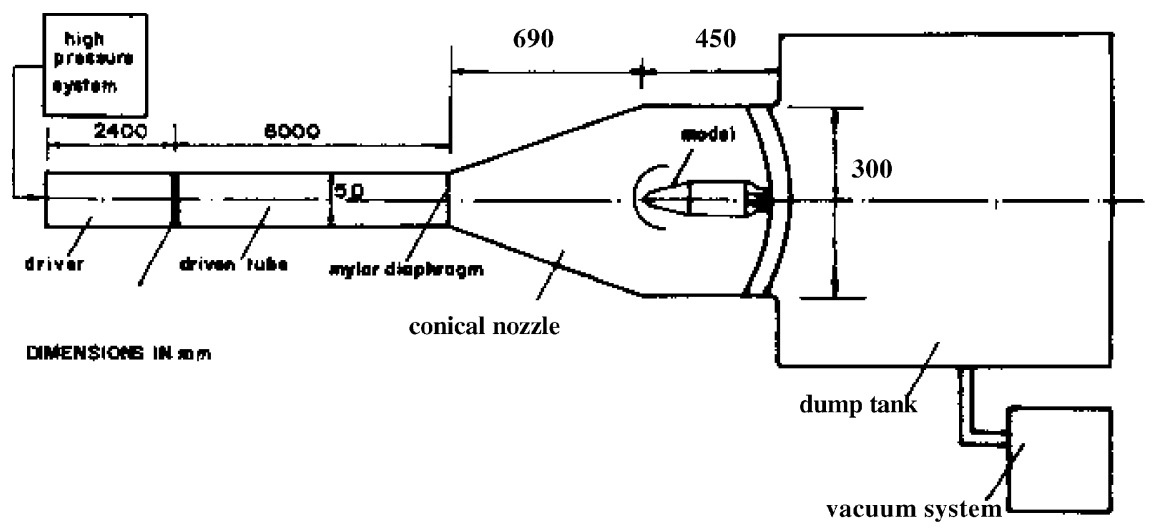

Fig. 2. Schematic diagram of IISc hypersonic shock tunnel HST2.

Table 1. Nominal flow conditions for experiments.

\begin{tabular}{ccccccccccc}
\hline Driver gas & $\begin{array}{c}H_{\mathrm{o}}{ }^{\mathrm{a}} \\
(\mathrm{MJ} / \mathrm{kg})\end{array}$ & $\begin{array}{c}P_{\mathrm{o}} \\
\left(\mathrm{KN} / \mathrm{m}^{2}\right)\end{array}$ & $\begin{array}{c}T_{\mathrm{o}} \\
(\mathrm{K})\end{array}$ & $\begin{array}{c}U_{\infty}{ }^{\mathrm{b}} \\
(\mathrm{m} / \mathrm{s})\end{array}$ & $\begin{array}{c}T_{\infty} \\
(\mathrm{K})\end{array}$ & $\begin{array}{c}\rho_{\infty} \\
\left(\mathrm{kg} / \mathrm{m}^{3}\right)\end{array}$ & $\begin{array}{c}P_{\infty} \\
\left(\mathrm{kN} / \mathrm{m}^{2}\right)\end{array}$ & $\begin{array}{c}M_{\infty} \\
R e \times 10^{6} \\
\left(\mathrm{~m}^{-1}\right)\end{array} \begin{array}{c}Q_{\mathrm{o}}{ }^{\mathrm{c}} \\
\left(\mathrm{W} / \mathrm{cm}^{2}\right)\end{array}$ \\
\hline Helium & 1.215 & 811.13 & 1209.56 & 1453.06 & 158.9 & 0.0146 & 666.4 & 5.75 & 1.965 & 28.7 \\
\hline
\end{tabular}

${ }^{a}$ Subscript $\mathrm{O}$ indicates reservoir condition. ${ }^{\mathrm{b}}$ Subscript $\infty$ denotes free stream conditions. ${ }^{\mathrm{c}}$ Measured stagnation point heating rate.

parameters. ${ }^{5)}$ In this study, a novel flow visualization technique $^{6)}$ based on an electrical discharge technique is used for visualizing flow around the blunt cone and measuring the shock standoff distance.

The main objectives of the present study: 1) Measurement of shock standoff distance around a $120^{\circ}$ blunt cone exposed to carbon dioxide and air streams at Mach 5.75 using electrical discharge technique, 2) Fore body convective heat transfer measurements around the blunt cone with both carbon dioxide and air as test gases, using platinum thin film sensors, and 3) Comparison of experimentally determined shock standoff distance with numerical simulation using the axisymmetric Euler solver VAS2D (Vectorized Adoptive Solver with 2 Spatial Variables) developed by Sun. ${ }^{7)}$ The detailed experimental methodologies and important results, followed by conclusions, are presented in the subsequent sections.

\section{Experimental Study}

Experiments were carried out in the recently constructed hypersonic shock tunnel HST2, which is capable of simulating stagnation enthalpy $\sim 5 \mathrm{MJ} / \mathrm{kg}$; this is schematically shown in Fig. 2. It comprises of a non-magnetic stainless steel shock tube $(50 \mathrm{~mm}$ internal diameter and $25 \mathrm{~mm}$ wall thickness) and wind tunnel sections. Typical blunt body model size of $100-150 \mathrm{~mm}$ diameter can be tested in the inviscid core of a $30 \times 30 \mathrm{~cm}$ test section. A Mach 6 conical nozzle is used for generating the hypersonic flow over the model by operating the tunnel in the straight-through mode. Based on the Pitot calibration runs, the Mach number of the test flow is estimated as 5.75 during the steady flow test time of $\sim 800 \mu \mathrm{s}$. Appropriate mechanical valves and fixtures are provided in the shock tube section for feeding any desired test gas. Helium and air are both used as driver gases. Typical experimental conditions are shown in Table 1. The flow visualization experiments are carried out using a $120^{\circ}$ blunt cone model with a base radius of $35 \mathrm{~mm}$ and a nose radius of $17.5 \mathrm{~mm}$ with slightly blunted (corner radius $=3 \mathrm{~mm}$ ) corner. For the heat transfer measurements a $120^{\circ}$ blunt cone model with base radius of $50 \mathrm{~mm}$, nose radius of $25 \mathrm{~mm}$ with slightly blunted (corner radius $=3 \mathrm{~mm}$ ) corner is used. Although a Bakelite hylem blunt cone model is used in visualization experiments, a blunt-cone model made of aluminium alloy along with Macor inserts is used in the heat transfer experiments. A schematic diagram of the blunt cone model and a photograph of the model used in the heat transfer measurements are shown in Fig. $3 a$ and $3 b$, respectively. The exact locations of the platinum thin-film sensors used in the surface convective heat transfer measurements are described in Table 2.

\subsection{Flow visualization experiments}

The intensity and spectral content of spontaneous light emitted in an electrical discharge depends on the density and temperature of the gas in the discharge path. Thus it is possible to visualize the shock shape and the gas temperature layer around bodies at hypersonic Mach numbers by generating an electric discharge over the model, then subsequently capturing the discharge light on photographic film. Nishio $^{8)}$ used the electrical discharge technique for visualizing the flow in hypersonic gun tunnels with typical test times of $10 \mathrm{~ms}$. Recently the flow field around an X-33 model has been visualized in a gun tunnel with this technique. ${ }^{9)}$ On the other hand the implementation of this technique in shock tunnels and in free-piston driven shock tunnels where both 
the test times and density levels are much lower is challenging.

The electrical discharge technique for visualizing shock
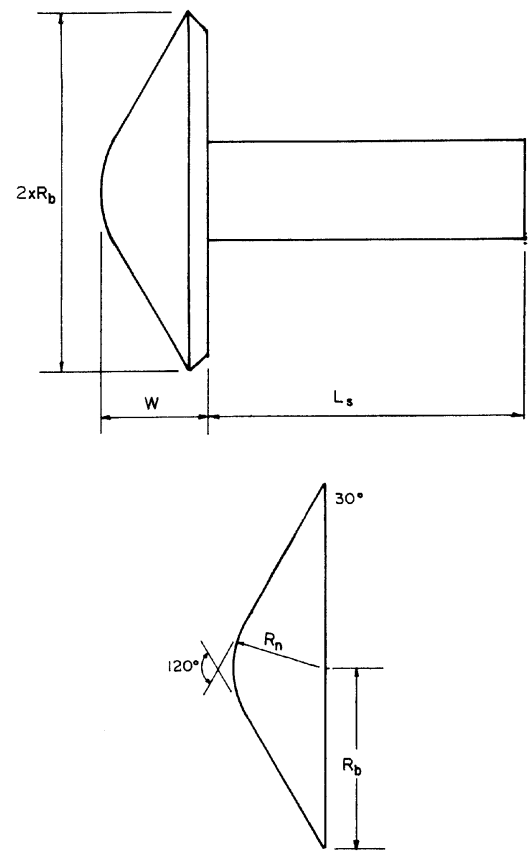

(a)

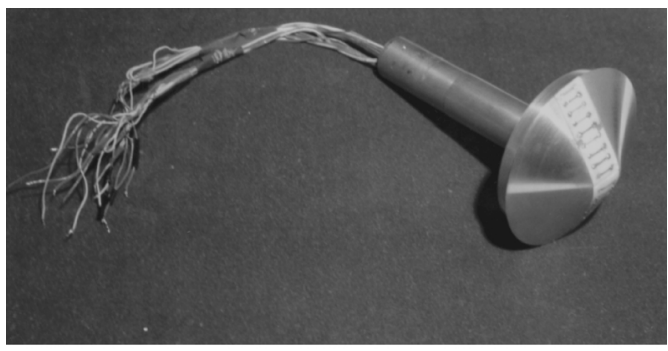

(b)

Fig. 3. (a) Schematic diagram of the blunt cone model $\left(R_{\mathrm{b}}=50\right.$; $\left.R_{\mathrm{n}} / R_{\mathrm{b}}=0.5 ; L_{\mathrm{s}}=150 ; W=35\right)$ used in the experiments. (b) Photograph of the blunt cone model used in the heat transfer studies.

Table 2. Location of platinum thin film sensors on the blunt cone.

\begin{tabular}{ccccccc}
\hline \multicolumn{3}{c}{ Windward side } & & \multicolumn{4}{c}{ Leeward side } \\
\cline { 1 - 3 } \cline { 5 - 7 } Gauge No. & $s^{\mathrm{a}}(\mathrm{mm})$ & $s / R_{\mathrm{n}}$ & & Gauge No. & $s(\mathrm{~mm})$ & $s / R_{\mathrm{n}}$ \\
\hline 1 & 0 & 0 & 1 & 0 & 0 \\
2 & 6 & 0.24 & 2 & -6 & -0.24 \\
3 & 12 & 0.48 & 3 & -12 & -0.48 \\
4 & 18 & 0.72 & 4 & -18 & -0.72 \\
5 & 24 & 0.96 & 5 & -24 & -0.96 \\
6 & 30 & 1.20 & 6 & -30 & -1.20 \\
7 & 36 & 1.44 & 7 & -36 & -1.44 \\
8 & 42 & 1.68 & 8 & -42 & -1.68 \\
9 & 48 & 1.92 & 9 & -48 & -1.92 \\
\hline
\end{tabular}

${ }^{a}$ Arc length on the surface of the cone is measured from the stagnation point. shapes in hypersonic shock tunnels with typical test times of less than a millisecond has been developed by Jagadeesh et al. $^{6,10)}$ The electrical discharge is generated across a pair of point-line electrodes. A point electrode of $1 \mathrm{~mm}$ diameter is attached to the roof of the tunnel test section, and a copper strip $0.5 \mathrm{~mm}$ thick is embedded vertically in the blunt cone model so that one of its edges is flush with the top surface of the model. A typical electrode arrangement used for visualizing hypersonic flow around the blunt cone is shown in Fig. 4. The electric circuit used for generating and controlling the electrical discharge is shown in Fig. 5. Because of the short test time in the shock tunnel, it is essential to precisely control the electrical discharge. For this purpose a pulse delay control unit comprising an 8-bit 8031 microprocessor, a 2764 EPROM, and a couple of digital switches for adjusting the pulse delay and duration has been developed. The appropriate delay during the experiments is adjusted by the use of a trigger signal from the heat transfer sensor at the end of the shock tube. The duration of the discharge was maintained at $2 \mu \mathrm{s}$, and the light emitted by the electrical discharge was photographed with a Nikon FM camera with $30 \mathrm{~mm}$ F1.4 lens operated in bulb exposure mode with ASA
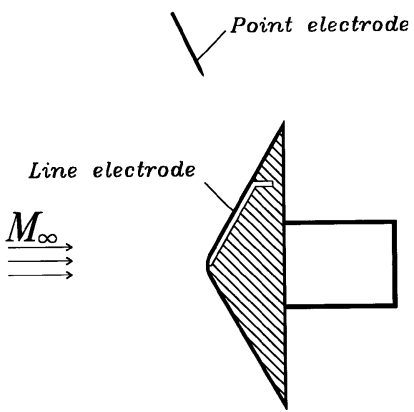

Fig. 4. Electrode arrangement in the flow visualization experiments using electrical discharge.

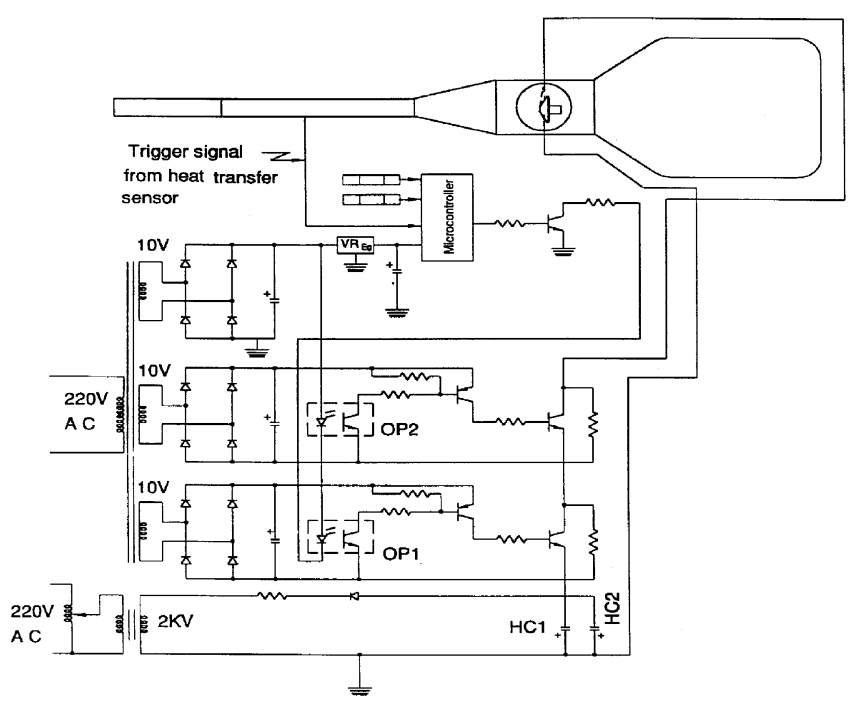

Fig. 5. Electric circuit used for the generation and control of electrical discharge. 
1600 film.

\subsection{Heat transfer measurements}

Platinum thin film sensors are deposited on ceramic glass Macor inserts, which are embedded in the metallic blunt cone model. The thin-film sensors are deposited on the Macor using platinum $05-\mathrm{X}$ metallo-organic ink $(\mathrm{M} / \mathrm{s}$ Englehard-Clal, UK). The sensors are connected through the analog network circuitry to the PC-based data acquisition system. The details of instrumentation used in heat transfer measurements along with the detailed data reduction methodology have been explained elsewhere. ${ }^{1)}$ The forebody convective heat transfer around the blunt cone is measured using both air and carbon dioxide as the test gases. When carbon dioxide is used as the test gas, initially vacuum is created in the driven section of the shock tube, and carbon dioxide is then allowed inside the tube. Because of the pressure differential between the residual air inside the shock tube and the carbon dioxide, the air is displaced through the exhaust of the vacuum pump. The process of vacuum creation followed by the operation of admitting carbon dioxide inside is repeated many times to ensure that the driven section is filled completely by carbon dioxide. Since the shock speed in carbon dioxide is lower compared to air, the driver/driven section pressure ratio is appropriately adjusted to achieve identical test conditions. In the present experiments only helium is used as the driver gas.

\subsection{Experimental uncertainties}

A conservative estimate of the error in the shock standoff measurements in air and carbon dioxide as the test gases is $\pm 5 \%$ and $\pm 7 \%$ respectively. Various factors such as shot to shot variation $( \pm 3 \%)$ in the flow conditions, the presence of traces of air in carbon dioxide runs, and uncertainties associated with the precise location of the blunt cone surface near the stagnation streamline because of slight masking of the blunt-cone surface by the bright discharge light near the line electrode in photographs contribute to the experimental errors. Since we are using copper line electrodes localized formation of oxide on the electrode results in bright spots during visualization and this will introduce uncertainties in locating the blunt cone surface. Further, the driven section of the shock tube is filled with carbon dioxide after repeatedly evacuating the tube. This is followed by the process of admitting carbon dioxide inside the tube. Even then, traces of air might be present in the shock tube during the run, and this also may introduce errors in the measured shock stand off distance. Based on the uncertainties associated with gauge characteristics, circuitry, data reduction techniques, and calibration, the measured values of heat transfer rates are believed to be accurate to $\pm 5 \%$ with helium as the driver gas. Various factors such as uncertainty in angle of attack, misalignment of Macro inserts, discontinuity in substrate and model properties, and departure from one-dimensional heat conduction during the run time contribute to uncertainties in the measured heat transfer values.

\section{Results and Discussion}

The visualized flow field around the blunt cone with air as the test gas is shown in Fig. 6. The detached shock wave is clearly seen, though the localized high intensity spot masks the shock wave near the corner of the blunt cone. A reduced distance between the point and the line electrode and thus least resistance for the electric discharge near the corner of the blunt cone are reasons for the appearance of such localized bright spots. Preliminary calibration experiments are carried out to isolate the effect of point electrode attachment interfering with the blunt cone flow field. The distance between the point-line electrodes is fixed at $50 \mathrm{~mm}$. The applied voltage between the electrodes is $1.65 \mathrm{kV}$. The platinum thin film heat transfer sensor near the end wall of the shock tube used to measure the speed of the shock wave is used to trigger the electrical discharge. The discharge photographs are scanned, and the digitized images are used for estimating the thickness of the shock layer near the stagnation streamline.

To order to complement the experimental study, the shock shape around the blunt cone is numerically simulated by solving axisymmetric Euler equations. The conservation laws are solved by the finite volume method on adaptive unstructured quadrilateral grids. The MUSCL-Hancock scheme ${ }^{11)}$ is used to determine the flux through interfaces. The gradients or slopes of primitive variables are calculated by the least square method. To maintain the TVD constraint the slopes are modified by the minmod limiter. The HLL approximate Riemann solver is chosen to determine the flux through interface based on two states that are respectively interpolated from two neighboring cells. For details on the numerical schemes, see reference. ${ }^{7)}$ Initially, a rather coarse grid that covers the whole computation domain is generated, and during computation the grid cells are locally divided around regions where flow variation is large, such as shock waves and slipstreams. In the computations, the initial background grid used for the calculation contains 1,659 cells, and this was increased to 13,209 cells by a three-level refinement when the final steady state is achieved. The computed shock shape around the blunt cone, along with the grid structure used in the numerical study, is shown in Fig. 7.

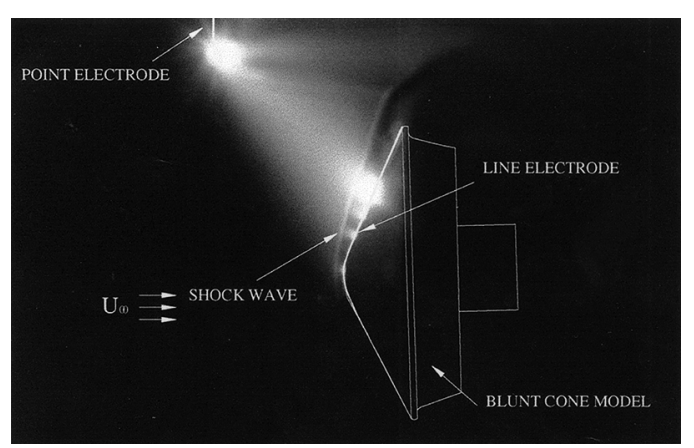

Fig. 6. The visualized flow field around a $120 \mathrm{deg}$. apex angle blunt cone at $M=5.75$. 


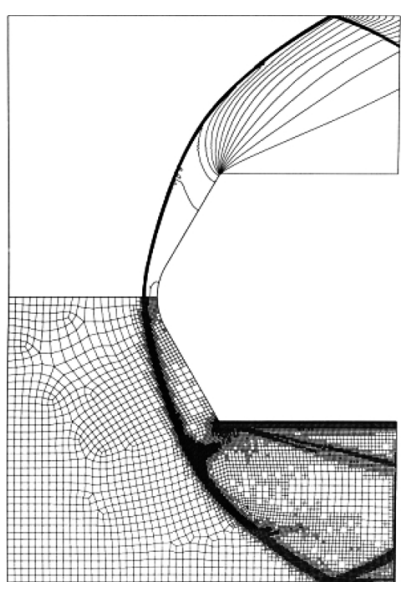

Fig. 7. The computed shock shape around the blunt cone with air as the test gas along with the typical grid structure used in the numerical study.

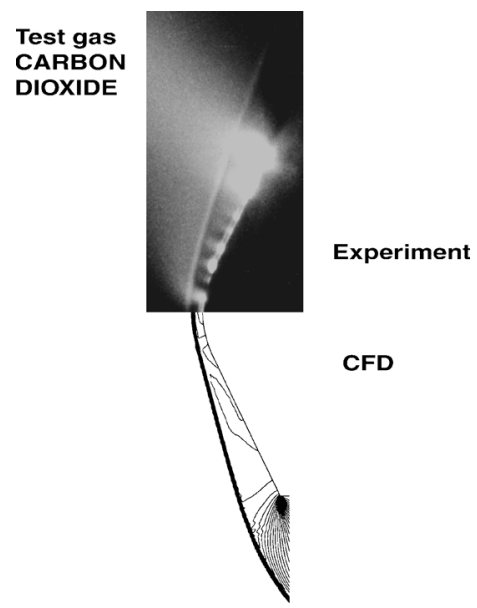

Fig. 8. A comparison between the computed and experimentally determined shock shape around the blunt cone with carbon dioxide as the test gas.

Both the experimentally visualized and the numerically simulated shock shapes around the blunt cone in a carbon dioxide environment are shown in Fig. 8. The experimentally determined shock layer thickness near the stagnation streamline is $3.29 \pm 0.26 \mathrm{~mm}$, and the numerically simulated thickness is $3.02 \mathrm{~mm}$. Figure 9 shows both the experimental and the numerically determined shock standoff distance on the blunt cone with both air and carbon dioxide as the test gas. The shock standoff distance on the blunt-cone model corresponds to the vertical ray expressed in terms of the wetted surface length $\left(s / R_{\mathrm{n}}\right)$ measured from the geometric stagnation point at zero angle of incidence. Although the agreement between experiments and computations are satisfactory near the stagnation zone, considerable deviations are observed away from the stagnation zone in a carbon dioxide environment. The computations are very sensitive to the value of $\delta$, which is strongly dependent on the temperature in carbon dioxide environment. Although the free stream temperature in the experiment is $\sim 150 \mathrm{~K}$, a $\gamma$ value of 1.328 (at $273 \mathrm{~K}$ ) is used in the computations. Experimentally deter-

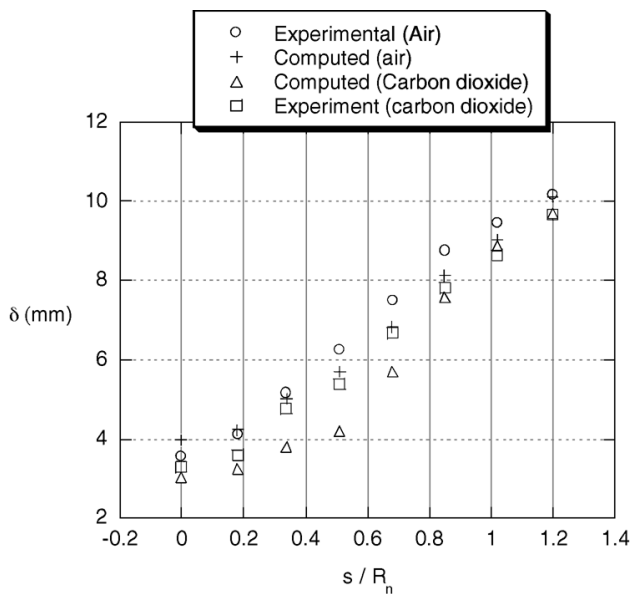

Fig. 9. Measured shock standoff distance around a blunt cone using carbon dioxide and air as test gases.

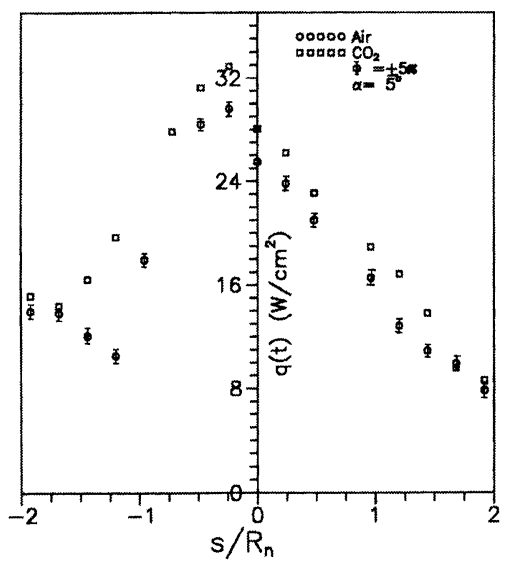

Fig. 10. The effects of test gas on the measured heat transfer rate around a blunt cone at an angle of incidence of $5^{\circ}$.

mined $\delta$ near the stagnation streamline with air as the test gas is $3.57 \pm 0.17 \mathrm{~mm}$, and the corresponding value in computation is $3.98 \mathrm{~mm}$. It should also be noted here that no real gas effects are included in the computations, since the dissociation effects are negligible in the experiments at a stagnation enthalpy of $1.2 \mathrm{MJ} / \mathrm{kg}$. Nevertheless, the overall agreement between experiment and computations is satisfactory.

Further convective heat transfer measurements were carried out to verify the reduced thickness of the shock layer in a carbon dioxide environment. With the reduced shock layer thickness and therefore with increased proximity of the bow shock to the blunt cone surface, the convective heat transfer should be higher in carbon dioxide than in air for the same flow conditions. The experimental heating rates on the blunt cone model correspond to the vertical ray expressed in terms of the wetted surface length $\left(s / R_{\mathrm{n}}\right)$ measured from the geometric stagnation point at zero angle of incidence. The effect of the test gas on the measured heat transfer on the blunt cone surface is shown in Fig. 10. An increase of 10 to $15 \%$ is observed with carbon dioxide as the test gas in comparison to air. These measurements are carried out at an angle of incidence of $5 \mathrm{deg}$. Although not shown here, 
the same trend is observed in the measurements regardless of angle of incidence. As expected, the windward side heat transfer increases with the increase in angle of attack from zero to 5 degrees. All these measurements are carried out at a stagnation enthalpy of $1.2 \mathrm{MJ} / \mathrm{kg}$.

Future study aims at creating a reliable experimental data bank of convective heating rates over large angle blunt cones in both continuum and rarefied hypersonic flow conditions with real gas effects. Concurrently, nonequilibrium computational codes will be developed to quantify the flow field around bodies of interest at hypervelocity flow regimes.

\section{Concluding Remarks}

The effect of carbon dioxide and air on the hypersonic flow field around a $120^{\circ}$ blunt cone is investigated. An electrical discharge technique was used for visualizing the flow field around the blunt cone at a zero angle of incidence. The fore body laminar convective heat transfer to the blunt cone was measured using platinum thin film sensors in air and carbon dioxide environments. An increase of 10 to $15 \%$ in the measured heat transfer values was observed with carbon dioxide as the test gas compared to air. The measured thickness of the shock layer along the stagnation streamline was $3.57 \pm 0.17 \mathrm{~mm}$ in air and $3.29 \pm 0.26 \mathrm{~mm}$ in carbon dioxide. The computed thickness of the shock layer for air and carbon dioxide were $3.98 \mathrm{~mm}$ and $3.02 \mathrm{~mm}$ respectively. The observed increase in the measured heat transfer rates in carbon dioxide compared to air was due to the higher density ratio across the bow shock wave and to the reduced shock layer thickness. Future work aims at creating an exhaustive data bank of reliable experimental heat transfer data for nu- merical code validation around large angle blunt cones.

\section{References}

1) Jagadeesh, G., Reddy, N. M., Nagashetty, K. and Reddy, K. P. J.: Fore Body Convective Heat Transfer Measurements over Large Angle Blunt Cones at Hypersonic Mach Number, AIAA Paper 98-2061, 1998.

2) Jagadeesh, G., Reddy, N. M., Nagashetty, K. and Reddy, K. P. J.: Fore Body Convective Hypersonic Heat Transfer Measurements over Large Angle Blunt Cones, J. Spacecraft Rockets, 37 (2000), pp. 137-139.

3) Stewart, D. A. and Chen, Y. K.: Hypersonic Convective Heat Transfer over 140-deg Blunt Cones in Different Gases, J. Spacecraft Rockets, 31 (1994), pp. 735-743.

4) Hollis, B. R. and Perkins, J. N.: High Enthalpy Aerothermodynamics of a Mars Entry Vehicle Part 1: Experimental Results, J. Spacecraft Rockets, 34 (1997), pp. 449-456.

5) Olivier, H.: A Theoretical Model for the Shock Stand-off Distance in Frozen and Equilibrium Flow, J. Fluid Mech., 413 (2000), pp. 345353.

6) Jagadeesh, G., Srinivasa, Rao, B. R., Nagashetty, K., Reddy, N. M. and Reddy, K. P. J.: A New Technique for Visualisation of Shock Shapes in Hypersonic Shock Tunnel, Curr. Sci. India, 71 (1996), pp. 128-130.

7) Sun, M.: Numerical and Experimental Studies of Shock Wave Interaction with Bodies, Ph. D. Thesis, Tohoku University, Japan, 1999.

8) Nishio, M.: New Method for Visualising Three-dimensional Shock Shapes around Hypersonic Vehicles Using an Electrical Discharge, AIAA J., 28 (1990), pp. 2085-2091.

9) Nishio, M. and Manabe, K.: Study on Flow Field around Hypersonic X-33 Model Utilising Electric Discharge Method, AIAA Paper 994958, 1999.

10) Jagadeesh, G., Srinivasa Rao, B. R., Nagashetty, K., Reddy, N. M. and Reddy, K. P. J.: Electrical Discharge Technique for Three-dimensional Visualisation in Hypersonic Shock Tunnel, J. Flow Visualisation and Image Processing, 4 (1997), pp. 51-57.

11) Toro, E. F.: Riemann Solvers and Numerical Methods for Fluid Dynamics, 2nd ed., Springer-Verlag, 1999. 